\title{
New dynamic critical phenomena in nuclear and quark superfluids
}

\author{
Noriyuki Sogabe and Naoki Yamamoto \\ Department of Physics, Keio University, Yokohama 223-8522, Japan
}

\begin{abstract}
We study the dynamic critical phenomena near the possible high-density QCD critical point inside the superfluid phase of nuclear and quark matter. We find that this critical point belongs to a new dynamic universality class beyond the conventional classification by Hohenberg and Halperin. We show that the speed of the superfluid phonon vanishes at the critical point and that the dynamic critical index is $z \approx 2$.
\end{abstract}

\section{INTRODUCTION}

Understanding the phase structure at finite temperature $T$ and baryon chemical potential $\mu$ is one of the important problems in quantum chromodynamics (QCD) 1]. Among others, it is an open issue to locate the landmark of the QCD phase diagram - the QCD critical point [2]. Theoretical analyses suggest the possible existence of the high-temperature critical point [3 7] between the hadron and quark-gluon plasma phases as well as high-density critical point [8 10] (see also Ref. [11]) between the hadron and color superconducting phases, as schematically illustrated in Fig. 11 However, not only their locations, but even their existences have not been established in QCD per se. This is mainly because firstprinciples lattice QCD calculations based on Monte Carlo sampling are not feasible due to the so-called sign problem. Therefore, it is important to provide theoretical predictions for their critical phenomena to be potentially tested in the beam energy scan program at the Relativistic Heavy Ion Collider (RHIC) and future experiments at the Facility of Antiproton and Ion Research (FAIR), Nuclotron-based Ion Collider Facility (NICA), and Japan Proton Accelerator Research Complex (J-PARC).

Generally, critical phenomena around critical points do not depend on the microscopic details of the systems, but only on the symmetries and low-energy degrees of freedom. This feature allows us to make model-independent predictions for critical phenomena. So far, theoretical

\footnotetext{
${ }^{1}$ In Refs. [8 10], the existence of the possible high-density critical point was found between the hadron phase and the so-called color-flavor locking phase [12]. In our discussion below, however, what is essential is the fact that high-density critical point is inside the superfluid phase with $\mathrm{U}(1)$ baryon number symmetry breaking (rather than it is inside the color superconducting phase); even if the chiral critical point exists inside the superfluid nuclear matter, our argument is applicable to it. For this reason, high-density region is described as the "nuclear/quark superfluid" in Fig. 1
}

studies of the QCD critical phenomena have been limited to the high-temperature critical point. It has been argued that its static universality class is that of the $3 \mathrm{D}$ Ising model [2], and the dynamic universality class is the so-called model $\mathrm{H}$ [13 16] in the classification by Hohenberg and Halperin [17]. On the other hand, the universality class of the high-density QCD critical point has not yet been elucidated until now.

In this paper, we study the static and dynamic universality classes of the high-density QCD critical point 2 We show that its static universality class is the same as that of the high-temperature QCD critical point. On the other hand, we find that its dynamic universality class is different from not only that of the high-temperature QCD critical point, but also those of all the models in the classification by Hohenberg and Halperin [17]; to the best of our knowledge, this is a new dynamic universality class that has not been found in any other system. As we will discuss in this paper, its uniqueness stems from the interplay between the chiral criticality and the presence of the superfluid phonon - a feature specific for the high-density QCD critical point. In other words, experimental identification of this unique dynamic critical phenomenon would provide indirect evidence of the superfluidity in the high-density regime of QCD.

This paper is organized as follows. In Sec. II, we identify a set of hydrodynamic variables near the highdensity QCD critical point. In Secs. III and IV] we study the static and dynamic critical phenomena near the high-density QCD critical point, respectively, by using the simplified model without the energy-momentum density. In Sec. V w we consider the full hydrodynamic

\footnotetext{
${ }^{2}$ Here, we will not consider the high-density critical point in the two-flavor color superconductivity in Ref. 11]. Because $\mathrm{U}(1)_{\mathrm{B}}$ symmetry is not broken in this phase [18], both of the static and dynamic universality classes are the same as those of the hightemperature critical point. Our focus in this paper will be on the high-density critical point with $\mathrm{U}(1)_{\mathrm{B}}$ symmetry breaking in Refs. 8, 9] (see also footnote 1).
} 


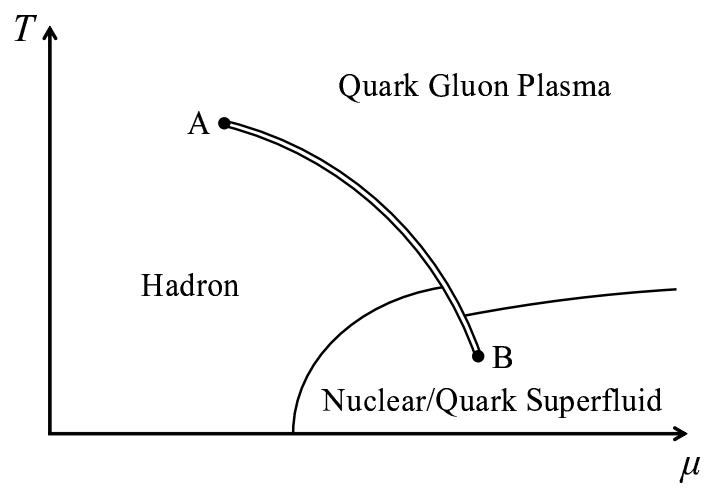

FIG. 1. A schematic QCD phase structure with the hightemperature critical point $\mathrm{A}$ and high-density critical point $\mathrm{B}$ [8, 9]. The double and single lines denote the first-order transition associated with chiral symmetry breaking/restoration and the second-order transition associated with $\mathrm{U}(1)_{\mathrm{B}}$ symmetry breaking/restoration, respectively.

modes and show that both the static and dynamic universality classes are the same as those in Secs. III and IV Finally, we conclude with Sec. VI, with the discussion on the physical reason why the high-density QCD critical point belong to the new dynamic universality class.

\section{HYDRODYNAMIC VARIABLES}

In general, critical phenomena near critical points at long distance and long time scale are independent of microscopic details and depend only on the low-energy degrees of freedom. To describe the critical phenomena of a given system, it is thus sufficient to consider the lowenergy effective theory for slow degrees of freedom called the hydrodynamic variables. Such a low-energy effective theory can be written down based on the systematic derivative expansion guided by the symmetries of the system. Typically, the hydrodynamic variables are the fluctuations of the conserved charges, the order parameter associated with the critical phenomena, and the NambuGoldstone modes associated with spontaneous breaking of some symmetries.

Near the high-density QCD critical point, the hydrodynamic variables are the fluctuations of the following quantities:

(i) The conserved energy and momentum densities, $\varepsilon \equiv T^{00}-\left\langle T^{00}\right\rangle$ and $\pi^{i} \equiv T^{0 i}$;

(ii) The conserved baryon number density, $n \equiv \bar{q} \gamma^{0} q-$ $\left\langle\bar{q} \gamma^{0} q\right\rangle$ (iii) The chiral condensate, $\sigma \equiv \bar{q} q-\langle\bar{q} q\rangle$;

(iv) The superfluid phonon $\theta$ associated with the spontaneous breaking of the $\mathrm{U}(1)_{\mathrm{B}}$ symmetry, $q \rightarrow q e^{i \theta}$.

The hydrodynamic variables (i)-(iii) above are the same as those of the high-temperature QCD critical point [15], but here we have the additional hydrodynamic variable (iv) due to the nuclear/quark superfluids.

Note that the fluctuation of the amplitude of the diquark condensate (which we denote by $\phi$ ) is not the hydrodynamic variable, because the high-density QCD critical point here is characterized by massless $\sigma$, and not by $\phi$; the high-density QCD critical point is not related to the second-order superfluid phase transition where $\phi$ becomes massless (see Fig. 11). Note also that NambuGoldstone modes associated with chiral symmetry breaking do not enter our low-energy effective theory because they acquire finite mass $m_{\pi}$ in the presence of finite quark masses; we are interested in the low-energy physics with the length scale much larger than $1 / m_{\pi}$. In the color superconducting phase, the gluons acquire the mass gap due to the color Meissner effect and do not enter the low-energy effective theory as well.

In the following, we will construct the low-energy effective theory describing the static and dynamic critical phenomena near the high-density QCD critical point, based on the symmetries of QCD at finite $T$ and $\mu$ : $\mathrm{U}(1)_{\mathrm{B}}$ symmetry, chiral symmetry, and discrete $\mathcal{C P} \mathcal{T}$ symmetries (charge conjugation, parity, and time reversal symmetry). Note that the time reversal symmetry is macroscopically broken by the presence of dissipation in the dynamical case.

As it will turn out that the energy and momentum densities, $\varepsilon$ and $\pi^{i}$, do not affect the static and dynamic critical phenomena, we will first consider the case only with the hydrodynamic variables $x_{i} \equiv \sigma, n, \theta$. We will then consider the case also with $\varepsilon$ and $\pi^{i}$ in Sec. $\mathrm{V}$ and show that they do not actually affect the static and dynamic universality classes.

\section{STATIC UNIVERSALITY CLASS}

Let us first study the static properties of the critical phenomena near the high-density QCD critical point. The general Ginzburg-Landau potential consistent with the QCD symmetries (with finite quark mass $m_{\mathrm{q}}$ and at finite chemical potential $\mu$ ) to the second order is given 
by

$$
\begin{aligned}
F[\sigma, n, \theta]=\int \mathrm{d} \boldsymbol{r}\left[\frac{a}{2}(\boldsymbol{\nabla} \sigma)^{2}\right. & +b \boldsymbol{\nabla} \sigma \cdot \boldsymbol{\nabla} n+\frac{c}{2}(\boldsymbol{\nabla} n)^{2} \\
& \left.+\frac{d}{2}(\boldsymbol{\nabla} \theta)^{2}+V(\sigma, n)\right],
\end{aligned}
$$

where

$$
V(\sigma, n)=\frac{A}{2} \sigma^{2}+B \sigma n+\frac{C}{2} n^{2} .
$$

Here the coefficients $a, b, c, d, A, B, C$ are the functions of $T$ and $\mu$ that depend on the microscopic details. Importantly, the mixing between $\sigma$ and $n$ is allowed in the presence of $m_{\mathrm{q}}$ and $\mu$ [15]. On the other hand, the mixing terms between $\theta$ and $\sigma$ or $n$ are prohibited by the time reversal symmetry in this static Ginzburg-Landau potential. (Note here that $\theta$ is $\mathcal{T}$ odd.)

One can calculate the correlation functions and static responses to external perturbations by

$$
\left\langle\mathcal{O}\left[x_{j}\right]\right\rangle=\frac{\int \prod_{i} \mathcal{D} x_{i} \mathcal{O}\left[x_{j}\right] e^{-\beta F}}{\int \prod_{i} \mathcal{D} x_{i} e^{-\beta F}} .
$$

Because $\theta$ is decoupled from $\sigma$ and $n$, the correlation function of $\sigma$ and the baryon number susceptibility defined by $\chi_{\mathrm{B}} \equiv \delta n / \delta \mu$ are the same as those for the hightemperature QCD critical point obtained in Ref. [15]:

$$
\begin{gathered}
\langle\sigma(\boldsymbol{r}) \sigma(\mathbf{0})\rangle=\frac{1}{4 \pi r} e^{-r / \xi}, \\
\chi_{\mathrm{B}}=\frac{1}{\mathcal{V} T}\left\langle n^{2}\right\rangle_{\boldsymbol{q} \rightarrow \mathbf{0}}=\frac{A}{\Delta},
\end{gathered}
$$

where $\mathcal{V}$ denotes the spatial volume and

$$
\xi \sim \Delta^{-\frac{1}{2}}, \quad \Delta \equiv A C-B^{2} .
$$

The critical point is characterized by the condition that the correlation length diverges, $\xi \rightarrow \infty$, or $\Delta \rightarrow 0$. Due to the mixing between $\sigma$ and $n$, there is only one linear combination of $\sigma$ and $n$ that becomes massless near the critical point [15]. Note here that $\theta$ is irrelevant to the condition for the criticality. Therefore, the static universality class of the high-density QCD critical point is the same as that of the high-temperature one obtained in Ref. 15]: the universality class of the 3D Ising model. Then, the critical exponent of $\chi_{\mathrm{B}}$ for the high-density QCD critical point can be obtained as

$$
\chi_{\mathrm{B}} \sim \xi^{2-\eta}
$$

with $\eta \simeq 0.04$.

\section{DYNAMIC UNIVERSALITY CLASS}

\section{A. Langevin equation}

We now discuss the dynamics of hydrodynamic variables near the high-density QCD critical point using the low-energy effective theory - the Langevin equation. Following the standard procedure (see, e.g., Ref. [19]), one can write down the generic Langevin theory for the hydrodynamic variables $x_{i}=\sigma, n, \theta$ as

$$
\begin{aligned}
\dot{\sigma}(\boldsymbol{r})= & -\Gamma \frac{\delta F}{\delta \sigma(\boldsymbol{r})}+\tilde{\lambda} \nabla^{2} \frac{\delta F}{\delta n(\boldsymbol{r})} \\
& -\int \mathrm{d} \boldsymbol{r}^{\prime}\left[\tilde{\sigma}(\boldsymbol{r}), \theta\left(\boldsymbol{r}^{\prime}\right)\right] \frac{\delta F}{\delta \theta\left(\boldsymbol{r}^{\prime}\right)}+\xi_{\sigma}(\boldsymbol{r}) \\
\dot{n}(\boldsymbol{r})= & \tilde{\lambda} \boldsymbol{\nabla}^{2} \frac{\delta F}{\delta \sigma(\boldsymbol{r})}+\lambda \boldsymbol{\nabla}^{2} \frac{\delta F}{\delta n(\boldsymbol{r})} \\
& -\int \mathrm{d} \boldsymbol{r}^{\prime}\left[\tilde{n}(\boldsymbol{r}), \theta\left(\boldsymbol{r}^{\prime}\right)\right] \frac{\delta F}{\delta \theta\left(\boldsymbol{r}^{\prime}\right)}+\xi_{n}(\boldsymbol{r}) \\
\dot{\theta}(\boldsymbol{r})= & -\int \mathrm{d} \boldsymbol{r}^{\prime}\left[\theta(\boldsymbol{r}), \tilde{\sigma}\left(\boldsymbol{r}^{\prime}\right)\right] \frac{\delta F}{\delta \sigma\left(\boldsymbol{r}^{\prime}\right)} \\
& -\int \mathrm{d} \boldsymbol{r}^{\prime}\left[\theta(\boldsymbol{r}), \tilde{n}\left(\boldsymbol{r}^{\prime}\right)\right] \frac{\delta F}{\delta n\left(\boldsymbol{r}^{\prime}\right)}-\zeta \frac{\delta F}{\delta \theta(\boldsymbol{r})}+\xi_{\theta}(\boldsymbol{r}) .
\end{aligned}
$$

Here $\Gamma, \lambda$, and $\tilde{\lambda}$ are the kinetic coefficients related to dissipation, $\xi_{\sigma}, \xi_{n}$, and $\xi_{\theta}$ are the noise terms, and we defined $\tilde{\sigma} \equiv \bar{q} q$ and $\tilde{n} \equiv \bar{q} \gamma^{0} q$. [On the other hand, note again that $\sigma$ and $n$ are defined as the fluctuations around the expectation values, $\sigma(\boldsymbol{r})=\tilde{\sigma}(\boldsymbol{r})-\sigma_{\mathrm{eq}}$ and $\left.n(\boldsymbol{r})=\tilde{n}(\boldsymbol{r})-n_{\mathrm{eq}} \cdot\right]$ The angler brackets denote the Poisson brackets, which we postulate as

$$
\begin{aligned}
& {\left[\tilde{\sigma}(\boldsymbol{r}), \theta\left(\boldsymbol{r}^{\prime}\right)\right]=0,} \\
& {\left[\theta(\boldsymbol{r}), \tilde{n}\left(\boldsymbol{r}^{\prime}\right)\right]=\delta\left(\boldsymbol{r}-\boldsymbol{r}^{\prime}\right) .}
\end{aligned}
$$

Equation (12) shows that $n$ is canonical conjugate to $\theta$. This can be understood from the $\mathrm{U}(1)$ gauge symmetry, which dictates that the Lagrangian for the superfluid phonon depends on $\theta$ through the form $\mathcal{L}=\mathcal{L}(\dot{\theta}+\mu, \nabla \theta)$ 20, 21], so that

$$
\tilde{n} \equiv \frac{\delta \mathcal{L}}{\delta \mu}=\frac{\delta \mathcal{L}}{\delta \dot{\theta}} .
$$

Some remarks on the Langevin equations above are in order here. The coefficients of the first two terms on the right-hand side of Eq. (9) include derivatives to satisfy the baryon number conservation, $\dot{n}=0$, in the long wavelength limit, $\boldsymbol{q} \rightarrow \mathbf{0}$. According to Onsager's principle, the coefficient of the second term in Eq. (8) must be the same as that of the first term in Eq. (9). The correlators of $\xi_{\sigma}, \xi_{n}$, and $\xi_{\theta}$ are chosen such that the equilibrium 
distribution $e^{-\beta F}$ is reproduced. Because the noise terms are not important at the mean-field level, we will ignore them in the following.

The variations $\delta F / \delta x_{i}$ can be computed by using the Ginzburg-Landau potential (11) as

$$
\begin{aligned}
& \frac{\delta F}{\delta \sigma}=\left(A-a \nabla^{2}\right) \sigma+\left(B-b \nabla^{2}\right) n, \\
& \frac{\delta F}{\delta n}=\left(B-b \nabla^{2}\right) \sigma+\left(C-c \nabla^{2}\right) n, \\
& \frac{\delta F}{\delta \theta}=-d \nabla^{2} \theta .
\end{aligned}
$$

By substituting Eqs. (11)-(15) into Eqs. (8)-(10) in momentum space $(\omega, \boldsymbol{q})$, the Langevin equation to the order of $O\left(\boldsymbol{q}^{2}\right)$ is summarized in the matrix form,

$$
\mathcal{M} \boldsymbol{x} \equiv\left(\begin{array}{ccc}
i \omega-\Gamma A-(\Gamma a+\tilde{\lambda} B) \boldsymbol{q}^{2} & -\Gamma B-(\Gamma b+\tilde{\lambda} C) \boldsymbol{q}^{2} & 0 \\
-(\tilde{\lambda} A+\lambda B) \boldsymbol{q}^{2} & i \omega-(\tilde{\lambda} B+\lambda C) \boldsymbol{q}^{2} & d \boldsymbol{q}^{2} \\
-B-b \boldsymbol{q}^{2} & -C-c \boldsymbol{q}^{2} & i \omega-\zeta d \boldsymbol{q}^{2}
\end{array}\right)\left(\begin{array}{c}
\sigma \\
n \\
\theta
\end{array}\right)=0
$$

\section{B. Hydrodynamic modes}

The hydrodynamic modes can be obtained by solving the proper equation, $\operatorname{det} \mathcal{M}=0$. This equation reduces to

$$
\omega^{3}+i\left(x_{1}+x_{2} \boldsymbol{q}^{2}\right) \omega^{2}-y \boldsymbol{q}^{2} \omega-i z \boldsymbol{q}^{2}=0,
$$

where

$$
\begin{aligned}
x_{1} & \equiv \Gamma A, \\
x_{2} & \equiv \Gamma a+2 \tilde{\lambda} B+\lambda C+\zeta d, \\
y & \equiv \Gamma \lambda \Delta+C d+\Gamma \zeta A d, \\
z & \equiv \Gamma d \Delta .
\end{aligned}
$$

In Eq. (17), we ignore the higher-order terms in $\boldsymbol{q}$. At this order, the left-hand side of Eq. (17) can be factorized as

$$
\begin{aligned}
& {\left[\omega+i x_{1}+i\left(x_{2}-\frac{y}{x_{1}}+\frac{z}{x_{1}^{2}}\right) \boldsymbol{q}^{2}+O\left(\boldsymbol{q}^{3}\right)\right] } \\
\times & {\left[\omega-\sqrt{\frac{z}{x_{1}}}|\boldsymbol{q}|+\frac{i}{2}\left(\frac{y}{x_{1}}-\frac{z}{x_{1}^{2}}\right) \boldsymbol{q}^{2}+O\left(\boldsymbol{q}^{3}\right)\right] } \\
\times & {\left[\omega+\sqrt{\frac{z}{x_{1}}}|\boldsymbol{q}|+\frac{i}{2}\left(\frac{y}{x_{1}}-\frac{z}{x_{1}^{2}}\right) \boldsymbol{q}^{2}+O\left(\boldsymbol{q}^{3}\right)\right]=0 . }
\end{aligned}
$$

This shows that the system near the high-density QCD critical point has three hydrodynamic modes: the relaxation mode and the pair of phonons with the dispersion relations,

$$
\begin{gathered}
\omega_{1}=-i \Gamma A+O\left(\boldsymbol{q}^{2}\right), \\
\omega_{2,3}= \pm c_{\mathrm{s}}|\boldsymbol{q}|+O\left(\boldsymbol{q}^{2}\right),
\end{gathered}
$$

respectively. Here

$$
c_{\mathrm{s}} \equiv \sqrt{\frac{d}{\chi_{\mathrm{B}}}}
$$

is the speed of the phonon.

We find that $c_{\mathrm{S}} \rightarrow 0$ due to the divergence of $\chi_{\mathrm{B}}$ when the critical point is approached [see Eq. (7)]. This is the phenomenon of the critical slowing down.

\section{Dynamic critical exponent}

The dynamic critical phenomena can be characterized by the dynamic critical exponent $z$ defined by $\tau \sim \xi^{z}$, or

$$
c_{\mathrm{S}} \sim \xi^{1-z}
$$

To find the value of $z$, we use the following $\xi$ dependences of $d$ and $\chi_{\mathrm{B}}$ near the high-density QCD critical point:

$$
d \sim \xi^{0}, \quad \chi_{\mathrm{B}} \sim \xi^{2-\eta}
$$

$d$ is the stiffness parameter (or the "decay constant" for the superfluid phonon), and it does not depend on $\xi$ close to the high-density critical point away from the superfluid phase transition. The behavior of $\chi_{\mathrm{B}}$ near the critical point is given by Eq. (77).

From Eqs. (22), (23), and (24), one finds the dynamic critical exponent $z$ as

$$
z=2-\frac{\eta}{2}
$$


This is different from those of the other models in the classification by Hohenberg and Halperin [17], indicating that the high-density QCD critical point belongs to a new dynamic universality class.

\section{COUPLING TO ENERGY AND MOMENTUM DENSITIES}

So far we have ignored the contributions of the energy and momentum densities, $\varepsilon$ and $\boldsymbol{\pi}$. In this section, we show that these contributions do not affect the static and dynamic universality classes of the high-density QCD critical point obtained above.

\section{A. Statics}

Let us first consider the Ginzburg-Landau functional to the second order in terms of $\sigma, n, \varepsilon, \boldsymbol{\pi}$, and $\theta$ describing static critical phenomena. Similarly to the argument in Sec. III. the time reversal symmetry prohibits the mixing between $x_{i} \equiv \sigma, n, \varepsilon$ and $\boldsymbol{\pi}, \theta$ :

$$
F[\sigma, n, \varepsilon, \boldsymbol{\pi}, \theta]=F[\sigma, n, \varepsilon]+F[\boldsymbol{\pi}, \theta] .
$$

The presence of $m_{\mathrm{q}}$ and $\mu$ generally allows the mixing among $\sigma, n$, and $\varepsilon$ in the $\mathcal{T}$-even sector as

$$
\begin{gathered}
F[\sigma, n, \varepsilon]=\frac{1}{2} \int \mathrm{d} \boldsymbol{r} x_{i} \beta_{i j}(\nabla) x_{j}, \\
\beta_{i j}(\boldsymbol{\nabla})=V_{i j}-v_{i j} \nabla^{2} .
\end{gathered}
$$

Here the subscripts $i, j$ are the shorthand notations for $x_{i}, x_{j}$. The $\mathcal{T}$-odd sector is given by

$$
F[\boldsymbol{\pi}, \theta]=\frac{1}{2} \int \mathrm{d} \boldsymbol{r}\left[V_{\pi \pi} \boldsymbol{\pi}^{2}+2 V_{\pi \theta} \boldsymbol{\pi} \cdot \boldsymbol{\nabla} \theta+V_{\theta \theta}(\boldsymbol{\nabla} \theta)^{2}\right] .
$$

We call $V_{i j}, v_{i j}, V_{\pi \pi}, V_{\pi \theta}$, and $V_{\theta \theta}$ the Ginzburg-Landau parameters.

By completing the square, Eq. (29) becomes

$$
F[\boldsymbol{\pi}, \theta]=\frac{1}{2} \int \mathrm{d} \boldsymbol{r}\left[V_{\pi \pi}\left(\boldsymbol{\pi}^{\prime}\right)^{2}+V_{\theta \theta}^{\prime}(\boldsymbol{\nabla} \theta)^{2}\right],
$$

where $\boldsymbol{\pi}^{\prime} \equiv \boldsymbol{\pi}+V_{\pi \theta} \boldsymbol{\nabla} \theta / V_{\pi \pi}$ and $V_{\theta \theta}^{\prime} \equiv V_{\theta \theta}-V_{\pi \theta}^{2} / V_{\pi \pi}$. Below we assume that $V_{\theta \theta}^{\prime}>0$ and redefine $\pi^{\prime}$ and $V_{\theta \theta}^{\prime}$ as $\pi$ and $V_{\theta \theta}$ for simplicity.

In a way similar to Eq. (4) in Sec. III the correlation length $\xi$ is defined from the correlation function of $\sigma$. In the present case, we find

$$
\xi \sim(\operatorname{det} V)^{-\frac{1}{2}}
$$

The critical point is characterized by the condition, $\operatorname{det} V=0$, analogously to the condition $\Delta=0$ in Sec. III. At the critical point, only one of the linear combinations of $\sigma, n$, and $\varepsilon$ becomes massless. Due to the degeneracy among $\sigma, n$, and $\varepsilon$, the static universality class remains the same as that of Sec. III.

We define the generalized susceptibilities

$$
\left.\chi_{i j} \equiv \frac{\delta\left\langle x_{i}\right\rangle_{X_{j}}}{\delta X_{j}}\right|_{X_{j}=0}
$$

where $\left\langle x_{i}\right\rangle_{X_{j}}$ is the the expectation value of $x_{i}$ in Eq. (3) with the replacement $\beta F \rightarrow \beta F+\int \mathrm{d} \boldsymbol{r} x_{j} X_{j}$ (where summation over the index $j$ is not implied) with $X_{\varepsilon} \equiv-\beta$, $X_{n} \equiv \beta \mu$, and $X_{\sigma} \equiv \beta m_{q}$. One can show the general relation between $\chi_{i j}$ and $V_{i j}$ as

$$
\begin{aligned}
\chi_{\varepsilon \varepsilon} & =\frac{1}{\mathcal{V}}\left\langle\varepsilon^{2}\right\rangle_{\boldsymbol{q} \rightarrow \mathbf{0}}=T\left(V^{-1}\right)_{\varepsilon \varepsilon}, \\
\chi_{\varepsilon n} & =\frac{1}{\mathcal{V}}\langle\varepsilon n\rangle_{\boldsymbol{q} \rightarrow \mathbf{0}}=T\left(V^{-1}\right)_{\varepsilon n}, \\
\chi_{n n} & =\frac{1}{\mathcal{V}}\left\langle n^{2}\right\rangle_{\boldsymbol{q} \rightarrow \mathbf{0}}=T\left(V^{-1}\right)_{n n},
\end{aligned}
$$

where $\left(V^{-1}\right)_{i j}$ denotes the $(i, j)$ component of the inverse matrix of $V$. Since $\left(V^{-1}\right)_{i j} \propto(\operatorname{det} V)^{-1}$, one finds

$$
\chi_{\varepsilon \varepsilon} \sim \chi_{\varepsilon n} \sim \chi_{n n} \sim \xi^{2-\eta},
$$

with $\eta \simeq 0.04$.

\section{B. Dynamics}

\section{Full Langevin equations}

To describe the dynamic critical phenomena, we now consider the Langevin equations in terms of the full hydrodynamic variables $x_{i}=\sigma, n, \varepsilon$, and $\pi, \theta$. The Langevin equations read

$$
\begin{aligned}
\dot{x}_{i}(\boldsymbol{r})= & -\gamma_{i j}(\boldsymbol{r}) \frac{\delta F}{\delta x_{j}(\boldsymbol{r})}-\int \mathrm{d} \boldsymbol{r}^{\prime}\left[\tilde{x}_{i}(\boldsymbol{r}), \boldsymbol{\pi}\left(\boldsymbol{r}^{\prime}\right)\right] \cdot \frac{\delta F}{\delta \boldsymbol{\pi}\left(\boldsymbol{r}^{\prime}\right)} \\
& -\int \mathrm{d} \boldsymbol{r}^{\prime}\left[\tilde{x}_{i}(\boldsymbol{r}), \theta\left(\boldsymbol{r}^{\prime}\right)\right] \frac{\delta F}{\delta \theta\left(\boldsymbol{r}^{\prime}\right)}+\xi_{i}(\boldsymbol{r}), \\
\dot{\boldsymbol{\pi}}(\boldsymbol{r})= & \Gamma_{\pi \pi} \boldsymbol{\nabla} \nabla \cdot \frac{\delta F}{\delta \boldsymbol{\pi}(\boldsymbol{r})}+\Gamma_{\pi \pi}^{\prime} \nabla^{2} \frac{\delta F}{\delta \boldsymbol{\pi}(\boldsymbol{r})}-\Gamma_{\pi \theta} \boldsymbol{\nabla} \frac{\delta F}{\delta \theta(\boldsymbol{r})} \\
& -\int \mathrm{d} \boldsymbol{r}^{\prime}\left[\boldsymbol{\pi}(\boldsymbol{r}), \tilde{x}_{i}\left(\boldsymbol{r}^{\prime}\right)\right] \frac{\delta F}{\delta x_{i}\left(\boldsymbol{r}^{\prime}\right)}+\boldsymbol{\xi}_{\pi}(\boldsymbol{r}), \\
\dot{\theta}(\boldsymbol{r})= & -\Gamma_{\pi \theta} \boldsymbol{\nabla} \cdot \frac{\delta F}{\delta \boldsymbol{\pi}(\boldsymbol{r})}-\Gamma_{\theta \theta} \frac{\delta F}{\delta \theta(\boldsymbol{r})} \\
& -\int \mathrm{d} \boldsymbol{r}^{\prime}\left[\theta(\boldsymbol{r}), \tilde{x}_{i}\left(\boldsymbol{r}^{\prime}\right)\right] \frac{\delta F}{\delta x_{i}\left(\boldsymbol{r}^{\prime}\right)}+\xi_{\theta}(\boldsymbol{r}),
\end{aligned}
$$


where summation over repeated indices $i, j$ is understood. Here

$$
\gamma_{i j} \equiv\left(\begin{array}{ccc}
\Gamma_{\sigma \sigma} & -\Gamma_{\sigma n} \nabla^{2} & -\Gamma_{\sigma \varepsilon} \nabla^{2} \\
-\Gamma_{\sigma n} \nabla^{2} & -\Gamma_{n n} \nabla^{2} & -\Gamma_{n \varepsilon} \nabla^{2} \\
-\Gamma_{\sigma \varepsilon} \nabla^{2} & -\Gamma_{n \varepsilon} \nabla^{2} & -\Gamma_{\varepsilon \varepsilon} \nabla^{2}
\end{array}\right),
$$

$\Gamma_{\alpha \beta}\left(\alpha, \beta=x_{i}, \pi, \theta\right)$, and $\Gamma_{\pi \pi}^{\prime}$ are the kinetic coefficients, and $\tilde{\varepsilon} \equiv T^{00}$. The noise terms $\xi_{i}, \boldsymbol{\xi}_{\pi}$, and $\xi_{\theta}$ above are not important in the following discussion. To write down the equations above, we took into account the momentum conservation law and the Onsager's principle, similarly to Sec. IV]

We postulate the Poisson brackets, in addition to Eqs. (11) and (12), as follows:

$$
\begin{aligned}
{\left[\boldsymbol{\pi}(\boldsymbol{r}), \tilde{\sigma}\left(\boldsymbol{r}^{\prime}\right)\right] } & =\tilde{\sigma}\left(\boldsymbol{r}^{\prime}\right) \boldsymbol{\nabla} \delta\left(\boldsymbol{r}-\boldsymbol{r}^{\prime}\right), \\
{\left[\boldsymbol{\pi}(\boldsymbol{r}), \tilde{n}\left(\boldsymbol{r}^{\prime}\right)\right] } & =\tilde{n}\left(\boldsymbol{r}^{\prime}\right) \nabla \delta\left(\boldsymbol{r}-\boldsymbol{r}^{\prime}\right), \\
{\left[\boldsymbol{\pi}(\boldsymbol{r}), \tilde{s}\left(\boldsymbol{r}^{\prime}\right)\right] } & =\tilde{s}\left(\boldsymbol{r}^{\prime}\right) \boldsymbol{\nabla} \delta\left(\boldsymbol{r}-\boldsymbol{r}^{\prime}\right), \\
{\left[\theta(\boldsymbol{r}), \tilde{s}\left(\boldsymbol{r}^{\prime}\right)\right] } & =0 .
\end{aligned}
$$

Here $\tilde{s}(\boldsymbol{r})$ denotes the entropy density. The Poisson brackets concerning $\tilde{\varepsilon}(\boldsymbol{r})$ can be derived as

$$
\begin{aligned}
{\left[\boldsymbol{\pi}(\boldsymbol{r}), \tilde{\varepsilon}\left(\boldsymbol{r}^{\prime}\right)\right] } & =(T \tilde{s}(\boldsymbol{r})+\mu \tilde{n}(\boldsymbol{r})) \boldsymbol{\nabla} \delta\left(\boldsymbol{r}-\boldsymbol{r}^{\prime}\right), \\
{\left[\theta(\boldsymbol{r}), \tilde{\varepsilon}\left(\boldsymbol{r}^{\prime}\right)\right] } & =\mu \delta\left(\boldsymbol{r}-\boldsymbol{r}^{\prime}\right) .
\end{aligned}
$$

Here, we used the thermodynamic relation, $\mathrm{d} \varepsilon=$ $T \mathrm{~d} s+\mu \mathrm{d} n$ and the definition of the Poisson brackets $\left[\boldsymbol{\pi}(\boldsymbol{r}), \tilde{y}_{i}\left(\boldsymbol{r}^{\prime}\right)\right] \equiv \delta \tilde{y}_{i}\left(\boldsymbol{r}^{\prime}\right) / \delta \boldsymbol{u}(\boldsymbol{r})$ for $\tilde{y}_{i} \equiv \tilde{n}, \tilde{s}, \tilde{\varepsilon}$, with $\boldsymbol{u}(\boldsymbol{r})$ the infinitesimal translation of the coordinate, $\boldsymbol{r} \rightarrow$ $\boldsymbol{r}+\boldsymbol{u}(\boldsymbol{r})$. (For the details of the Poisson brackets, see, e.g., Ref. 22].)

We consider the small fluctuations of variables around the equilibrium values, $\sigma(\boldsymbol{r})=\tilde{\sigma}(\boldsymbol{r})-\sigma_{\text {eq }}, n(\boldsymbol{r})=\tilde{n}(\boldsymbol{r})-$ $n_{\text {eq }}$, and $s(\boldsymbol{r})=\tilde{s}(\boldsymbol{r})-s_{\text {eq }}$. Then, the linearized Langevin equations can be written as follows:

$$
\begin{aligned}
\dot{\sigma}= & -\left(\Gamma_{\sigma \sigma} V_{\sigma i}-\Gamma_{\sigma j} v_{j i} \nabla^{2}\right) x_{i}-\sigma_{\mathrm{eq}} V_{\pi \pi} \boldsymbol{\nabla} \cdot \boldsymbol{\pi}, \\
\dot{n}= & \Gamma_{n j} v_{j i} \nabla^{2} x_{i}-n_{\mathrm{eq}} V_{\pi \pi} \boldsymbol{\nabla} \cdot \boldsymbol{\pi}-V_{\theta \theta} \nabla^{2} \theta, \\
\dot{\varepsilon}= & \Gamma_{\varepsilon j} v_{j i} \boldsymbol{\nabla}^{2} x_{i}-w_{\mathrm{eq}} V_{\theta \theta} \boldsymbol{\nabla} \cdot \boldsymbol{\pi}-\mu V_{\theta \theta} \boldsymbol{\nabla}^{2} \theta, \\
\dot{\boldsymbol{\pi}}= & -\left(\sigma_{\mathrm{eq}} V_{\sigma i}+n_{\mathrm{eq}} V_{n i}+w_{\mathrm{eq}} V_{\varepsilon i}\right) \boldsymbol{\nabla} x_{i} \\
& +\Gamma_{\pi \pi} V_{\pi \pi} \boldsymbol{\nabla} \boldsymbol{\nabla} \cdot \boldsymbol{\pi}+\Gamma_{\pi \pi}^{\prime} V_{\pi \pi} \boldsymbol{\nabla}^{2} \boldsymbol{\pi} \\
\dot{\theta}= & -\left[\left(V_{n i}+\mu V_{\varepsilon i}\right)-\left(v_{n i}+\mu v_{\varepsilon i}\right) \nabla^{2}\right] x_{i} \\
& -\Gamma_{\pi \theta} V_{\pi \pi} \boldsymbol{\nabla} \cdot \boldsymbol{\pi}+\Gamma_{\theta \theta} V_{\theta \theta} \boldsymbol{\nabla}^{2} \theta
\end{aligned}
$$

where $w_{\mathrm{eq}}=T s_{\mathrm{eq}}+\mu n_{\mathrm{eq}}$.

\section{Decomposition of momentum density}

It is convenient to work in the $(t, \boldsymbol{q})$ space to decompose the momentum density $\pi^{i}$ into the longitudinal and transverse parts with respect to momentum $\boldsymbol{q}$,

$$
\pi^{i}=\pi_{\mathrm{L}}^{i}+\pi_{\mathrm{T}}^{i}, \quad \pi_{\mathrm{L}}^{i}=\left(P_{\mathrm{L}}\right)^{i j} \pi^{j}, \quad \pi_{\mathrm{T}}^{i}=\left(P_{\mathrm{T}}\right)^{i j} \pi^{j},
$$

where $P_{\mathrm{L}, \mathrm{T}}$ are the longitudinal and transverse projections defined by

$$
\left(P_{\mathrm{L}}\right)^{i j} \equiv \frac{q^{i} q^{j}}{|\boldsymbol{q}|^{2}}, \quad\left(P_{\mathrm{T}}\right)^{i j} \equiv \delta_{i j}-\frac{q^{i} q^{j}}{|\boldsymbol{q}|^{2}} .
$$

One can show that the dynamics of $\pi_{\mathrm{T}}^{i}$ is decoupled from the dynamics of the other variables as follows. The linearized Langevin equations obtained above can be written down to the leading order of $\boldsymbol{q}$ as

$$
\begin{aligned}
\dot{x}_{k} & =A_{x_{k}}(\boldsymbol{q} \cdot \boldsymbol{\pi})+f\left(x_{k}, \theta\right), \\
\dot{\boldsymbol{\pi}} & =A_{\pi} \boldsymbol{q}(\boldsymbol{q} \cdot \boldsymbol{\pi})+B_{\pi}|\boldsymbol{q}|^{2} \boldsymbol{\pi}+\boldsymbol{q} g\left(x_{k}\right), \\
\dot{\theta} & =A_{\theta}(\boldsymbol{q} \cdot \boldsymbol{\pi})+h\left(x_{k}, \theta\right),
\end{aligned}
$$

where $f\left(x_{k}, \theta\right), g\left(x_{k}\right)$, and $h\left(x_{k}, \theta\right)$ denote the terms that may involve $x_{k}$ and $\theta$, but do not $\boldsymbol{\pi}$. The coefficients $A_{x_{k}}$, $A_{\pi}, A_{\theta}$, and $B_{\pi}$ denote the parameters which depend on the Ginzburg-Landau parameters $V_{i j}, v_{i j}$, kinetic coefficients, and equilibrium values of the thermodynamic quantities. The explicit forms of these coefficients are not important for our purpose. Using $\boldsymbol{\pi}_{\mathrm{L}, \mathrm{T}}$, Eqs. (54)-(56) can be rewritten as

$$
\begin{aligned}
\dot{x}_{k} & =A_{x_{k}}\left(\boldsymbol{q} \cdot \boldsymbol{\pi}_{\mathrm{L}}\right)+f\left(x_{k}, \theta\right), \\
\dot{\boldsymbol{\pi}}_{\mathrm{L}} & =\left(A_{\pi}+B_{\pi}\right)|\boldsymbol{q}|^{2} \boldsymbol{\pi}_{\mathrm{L}}+\boldsymbol{q} g\left(x_{k}\right), \\
\dot{\boldsymbol{\pi}}_{\mathrm{T}} & =B_{\pi}|\boldsymbol{q}|^{2} \boldsymbol{\pi}_{\mathrm{T}}, \\
\dot{\theta} & =A_{\theta}\left(\boldsymbol{q} \cdot \boldsymbol{\pi}_{\mathrm{L}}\right)+h\left(x_{k}, \theta\right) .
\end{aligned}
$$

So the dynamics of $\pi_{\mathrm{L}}^{i}$ and $\pi_{\mathrm{T}}^{i}$ are decoupled from each other at the mean-field level.

\section{Hydrodynamic modes}

It is easy to obtain the Langevin equation for $\boldsymbol{\pi}_{T}$ in the $(\omega, \boldsymbol{q})$ space as

$$
\left(i \omega-B_{\pi} \boldsymbol{q}^{2}\right) \boldsymbol{\pi}_{\mathrm{T}}=0, \quad B_{\pi}=\Gamma_{\pi \pi}^{\prime} V_{\pi \pi},
$$

which shows the diffusion mode.

The other Langevin equations which involve $\boldsymbol{\pi}_{\mathrm{L}}$ (but not $\boldsymbol{\pi}_{\mathrm{T}}$ ) can be summarized in the form of the matrix equation, 


$$
\mathcal{M}\left(\begin{array}{c}
x_{i} \\
\boldsymbol{\pi}_{\mathrm{L}} \\
\theta
\end{array}\right) \equiv\left(\begin{array}{rrrrr}
i \omega-A_{\sigma \sigma}-a_{\sigma \sigma} \boldsymbol{q}^{2} & -A_{\sigma n}-a_{\sigma n} \boldsymbol{q}^{2} & -A_{\sigma \varepsilon}-a_{\sigma \varepsilon} \boldsymbol{q}^{2} & -i a_{\sigma \pi} \boldsymbol{q} & 0 \\
-a_{n \sigma} \boldsymbol{q}^{2} & i \omega-a_{n n} \boldsymbol{q}^{2} & -a_{n \varepsilon} \boldsymbol{q}^{2} & -i a_{n \pi} \boldsymbol{q} & -a_{n \theta} \boldsymbol{q}^{2} \\
-a_{\varepsilon \sigma} \boldsymbol{q}^{2} & -a_{\varepsilon n} \boldsymbol{q}^{2} & i \omega-a_{\varepsilon \varepsilon} \boldsymbol{q}^{2} & -i a_{\varepsilon \pi} \boldsymbol{q} & -a_{\varepsilon \theta} \boldsymbol{q}^{2} \\
-i a_{\pi \sigma} \boldsymbol{q} & -i a_{\pi n} \boldsymbol{q} & -i a_{\pi \varepsilon} \boldsymbol{q} & i \omega-a_{\pi \pi} \boldsymbol{q}^{2} & 0 \\
-A_{\theta \sigma}-a_{\theta \sigma} \boldsymbol{q}^{2} & -A_{\theta n}-a_{\theta n} \boldsymbol{q}^{2} & -A_{\theta \varepsilon}-a_{\theta \varepsilon} \boldsymbol{q}^{2} & -i a_{\theta \pi} \boldsymbol{q} & i \omega-a_{\theta \theta} \boldsymbol{q}^{2}
\end{array}\right)\left(\begin{array}{c}
\sigma \\
n \\
\varepsilon \\
\boldsymbol{\pi}_{\mathrm{L}} \\
\theta
\end{array}\right)=0
$$

where $A_{\alpha \beta}$ and $a_{\alpha \beta}$ are the parameters depending on $V_{i j}, v_{i j}$, kinetic coefficients, and thermodynamic quantities. Note that $A_{\alpha \beta}, a_{\alpha \beta}$ are not symmetric with respect to $\alpha$ and $\beta$.

The eigenfrequencies of Eq. (62) can be found from $\operatorname{det} \mathcal{M}=0$, which yields

$$
\begin{aligned}
\omega^{5}+i\left(A_{\sigma \sigma}+g_{4} \boldsymbol{q}^{2}\right) \omega^{4}- & {\left[g_{3} \boldsymbol{q}^{2}+O\left(\boldsymbol{q}^{4}\right)\right] \omega^{3} } \\
-i\left[g_{2} \boldsymbol{q}^{2}+O\left(\boldsymbol{q}^{4}\right)\right] \omega^{2}+\left[g_{1} \boldsymbol{q}^{4}+O\left(\boldsymbol{q}^{6}\right)\right] \omega & \\
+i\left[g_{0} \boldsymbol{q}^{4}+O\left(\boldsymbol{q}^{6}\right)\right]=0 &
\end{aligned}
$$

where $g_{0}, \cdots, g_{4}$ are the functions of $A_{\alpha \beta}$ and $a_{\alpha \beta}$. Among others, we only give the explicit expressions for $A_{\sigma \sigma}, g_{2}$, and $g_{0}$,

$$
\begin{aligned}
A_{\sigma \sigma} \equiv & \Gamma_{\sigma \sigma}, \\
g_{2} \equiv & \Gamma_{\sigma \sigma}\left(n_{\mathrm{eq}}^{2} V_{\pi \pi}+V_{\theta \theta}\right)\left|\begin{array}{cc}
V_{\sigma \sigma} & V_{\sigma n} \\
V_{\sigma n} & V_{n n}
\end{array}\right| \\
& +2 \Gamma_{\sigma \sigma}\left(n_{\mathrm{eq}} w_{\mathrm{eq}} V_{\pi \pi}+\mu V_{\theta \theta}\right)\left|\begin{array}{cc}
V_{\sigma \sigma} & V_{\sigma n} \\
V_{\sigma \varepsilon} & V_{n \varepsilon}
\end{array}\right| \\
& +\Gamma_{\sigma \sigma}\left(w_{\mathrm{eq}}^{2} V_{\pi \pi}+\mu^{2} V_{\theta \theta}\right)\left|\begin{array}{cc}
V_{\sigma \sigma} & V_{\sigma \varepsilon} \\
V_{\sigma \varepsilon} & V_{\varepsilon \varepsilon}
\end{array}\right|, \\
g_{0} \equiv & \Gamma_{\sigma \sigma} V_{\pi \pi} V_{\theta \theta} T^{2} s_{\mathrm{eq}}^{2} \operatorname{det} V,
\end{aligned}
$$

which will be used in the following discussion.

Equation (63) can be factorized as

$$
\begin{aligned}
& {\left[\omega+i A_{\sigma \sigma}+i\left(g_{4}-\frac{g_{3}}{A_{\sigma \sigma}}+\frac{g_{2}}{A_{\sigma \sigma}^{2}}\right) \boldsymbol{q}^{2}\right]} \\
& \times\left[\omega-s_{+}|\boldsymbol{q}|+i \frac{t_{+}}{2} \boldsymbol{q}^{2}\right]\left[\omega+s_{+}|\boldsymbol{q}|+i \frac{t_{+}}{2} \boldsymbol{q}^{2}\right] \\
& \times\left[\omega-s_{-}|\boldsymbol{q}|+i \frac{t_{-}}{2} \boldsymbol{q}^{2}\right]\left[\omega+s_{-}|\boldsymbol{q}|+i \frac{t_{-}}{2} \boldsymbol{q}^{2}\right]=0,
\end{aligned}
$$

where $s_{ \pm}$and $t_{ \pm}$satisfy

$$
\begin{aligned}
s_{+}^{2}+s_{-}^{2} & =\frac{g_{2}}{A_{\sigma \sigma}}, \\
s_{+}^{2} s_{-}^{2} & =\frac{g_{0}}{A_{\sigma \sigma}}, \\
t_{+}+t_{-} & =\frac{g_{3}}{A_{\sigma \sigma}}-\frac{g_{2}}{A_{\sigma \sigma}^{2}}, \\
s_{+}^{2} t_{-}+s_{-}^{2} t_{+} & =\frac{g_{1}}{A_{\sigma \sigma}}-\frac{g_{0}}{A_{\sigma \sigma}^{2}} .
\end{aligned}
$$

From Eq. 676), one finds all the hydrodynamic modes, except for the diffusion mode described by Eq. (61): one relaxation mode and two pairs of phonons. The speeds of phonons, $s_{ \pm}$, can be obtained from the solution of

$$
s^{4}-\frac{g_{2}}{A_{\sigma \sigma}} s^{2}+\frac{g_{0}}{A_{\sigma \sigma}}=0 .
$$

Note here that Eq. (66) shows that $g_{0} \rightarrow 0$ as the critical point is approached, $\operatorname{det} V \rightarrow 0$. Near the critical point, we thus obtain

$$
s_{+}^{2}=\frac{g_{2}}{A_{\sigma \sigma}}-\frac{g_{0}}{g_{2}}, \quad s_{-}^{2}=\frac{g_{0}}{g_{2}} .
$$

\section{Dynamic critical exponent}

From the results above, we can show that one of the phonons with the speed $c_{\mathrm{S}} \equiv s_{-}$exhibits the critical slowing down as follows. By using Eqs. (65) and (66), together with Eqs. (33)- (35), we have

$$
c_{\mathrm{s}}^{2}=\frac{V_{\pi \pi} V_{\theta \theta} T^{3} s_{\mathrm{eq}}^{2}}{\kappa_{n n} \chi_{n n}+2 \kappa_{n \varepsilon} \chi_{n \varepsilon}+\kappa_{\varepsilon \varepsilon} \chi_{\varepsilon \varepsilon}},
$$

where

$$
\begin{aligned}
\kappa_{n n} & \equiv n_{\mathrm{eq}}^{2} V_{\pi \pi}+V_{\theta \theta}, \\
\kappa_{n \varepsilon} & \equiv n_{\mathrm{eq}} w_{\mathrm{eq}} V_{\pi \pi}+\mu V_{\theta \theta}, \\
\kappa_{\varepsilon \varepsilon} & \equiv w_{\mathrm{eq}}^{2} V_{\pi \pi}+\mu^{2} V_{\theta \theta} .
\end{aligned}
$$

Using Eq. (36), $V_{\pi \pi} \sim \xi^{0}$, and $V_{\theta \theta} \sim \xi^{0}$ near the critical point, we obtain

$$
c_{\mathrm{s}}^{2} \sim \xi^{-2+\eta}
$$


Comparing it with Eq. (23), we find that the dynamic critical exponent $z$ is again given by Eq. (25): the dynamic universality class remains the same as the case without the coupling to $\varepsilon$ and $\boldsymbol{\pi}$ in Sec. IV

Finally, we remark that the dynamic critical exponent $z$ obtained above is not affected by nonlinear couplings beyond the mean field. This can be understood by recalling that what is renormalized by nonlinear terms is the kinetic coefficient [19] and that Eq. (74) does not depend on any kinetic coefficient. This should be contrasted with the case of the high-temperature QCD critical point, where the dynamic critical exponent is modified by nonlinear couplings [15]. This is because the hydrodynamic mode that exhibits the critical slowing down there is the diffusion mode, whose diffusion rate depends on a kinetic coefficient.

\section{CONCLUSION AND DISCUSSION}

In this paper, we have shown that the high-density QCD critical point belongs to the new dynamic universality class that is beyond the conventional classification by Hohenberg and Halperin. We have demonstrated that the speed of the superfluid phonon vanishes as the critical point is approached and that the dynamic critical index is $z \approx 2$.

The physical reason why the dynamic universality class of the high-density QCD critical point is new can be understood as follows. First, it is different from that of the high-temperature QCD critical point (i.e., model $\mathrm{H}$ [15]) due to the presence of the superfluid phonon associated with the $\mathrm{U}(1)_{\mathrm{B}}$ symmetry breaking. Second, it must also be different from that of the superfluid transition of ${ }^{4} \mathrm{He}$ despite the presence of superfluid phonons in both cases. This is because the former criticality is charac- terized by the vanishing chiral order parameter (with a nonzero superfluid gap), while the latter is characterized by the vanishing superfluid gap (or vanishing stiffness constant) 3

Our results suggest that, while the static critical phenomena cannot distinguish between the hightemperature and high-density QCD critical points, the dynamic critical phenomena can distinguish between the two. It would be important to study possible phenomenological consequences and experimental signatures of the high-density critical point to be tested in future heavy ion collision experiments, similarly to the high-temperature one 23]. The uniqueness of the dynamic critical phenomena around the high-density QCD critical point, if observed, would provide indirect evidence of the superfluidity in high-density QCD matter.

Finally, it would also be interesting to study the possible effects of dynamical electromagnetic fields (or massless photons), which may affect not only the dynamic universality class of the high-density critical point, but also even that of the high-temperature one.

\section{ACKNOWLEDGMENTS}

We thank H. Fujii and Y. Hidaka for useful discussions. This work was supported by JSPS KAKENHI Grant No. 16K17703 and MEXT-Supported Program for the Strategic Research Foundation at Private Universities, "Topological Science" (Grant No. S1511006). N. S. acknowledges the Research Grant of Keio Leading-edge Laboratory of Science and Technology and the fellowship of the European Physical Society (EPS) provided through the International School of Nuclear Physics 2016 in Erice, where this work was completed.

\footnotetext{
${ }^{3}$ In fact, this difference of order parameters is reflected in the difference of the critical behaviors of the speeds of superfluid phonons between the two cases. In the superfluid ${ }^{4} \mathrm{He}$, the speed of superfluid phonon is given by $c_{\mathrm{S}}=\sqrt{\rho_{\mathrm{s}} / c_{\mathrm{p}}}$ [17], where $\rho_{\mathrm{S}}$ is the stiffness constant and $c_{\mathrm{p}}$ is the specific heat at constant pressure. When the superfluid transition is approached (i.e., when the correlation length diverges, $\xi \rightarrow \infty$ ), the stiffness constant goes to zero as $\rho_{\mathrm{s}} \sim \xi^{-1}$ and $c_{\mathrm{p}} \sim \xi^{\alpha / \nu}$. [Here, $\alpha$ and $\nu$ are the critical exponents of $c_{\mathrm{p}}$ and $\xi$, defined as $c_{\mathrm{p}} \sim \tau^{-\alpha}$ and $\xi \sim \tau^{-\nu}$ with $\tau \equiv\left(T-T_{\mathrm{c}}\right) / T_{\mathrm{c}}$.] In particular, the $\xi$ dependence of $c_{\mathrm{S}}$
}

[1] K. Fukushima and T. Hatsuda, Rep. Prog. Phys. 74, 014001 (2011).

[2] M. A. Stephanov, Prog. Theor. Phys. Suppl. 153, 139 (2004) [Int. J. Mod. Phys. A 20, 4387 (2005)].

[3] M. Asakawa and K. Yazaki, Nucl. Phys. A504, 668 (1989).

is different from Eq. (22), because the high-density QCD critical point is away from the superfluid transition and the stiffness parameter $d$ in Eq. (1) remains nonvanishing. 
[4] A. Barducci, R. Casalbuoni, S. De Curtis, R. Gatto, and G. Pettini, Phys. Lett. B 231, 463 (1989).

[5] A. M. Halasz, A. D. Jackson, R. E. Shrock, M. A. Stephanov, and J. J. M. Verbaarschot, Phys. Rev. D 58, 096007 (1998).

[6] J. Berges and K. Rajagopal, Nucl. Phys. B538, 215 (1999).

[7] Y. Hatta and T. Ikeda, Phys. Rev. D 67, 014028 (2003).

[8] T. Hatsuda, M. Tachibana, N. Yamamoto, and G. Baym, Phys. Rev. Lett. 97, 122001 (2006); Phys. Rev. D 76, 074001 (2007).

[9] H. Abuki, G. Baym, T. Hatsuda, and N. Yamamoto, Phys. Rev. D 81, 125010 (2010).

[10] A. Schmitt, S. Stetina, and M. Tachibana, Phys. Rev. D 83, 045008 (2011).

[11] M. Kitazawa, T. Koide, T. Kunihiro, and Y. Nemoto, Prog. Theor. Phys. 108, 929 (2002).

[12] M. G. Alford, K. Rajagopal, and F. Wilczek, Nucl. Phys. B537, 443 (1999).

[13] H. Fujii, Phys. Rev. D 67, 094018 (2003).

[14] H. Fujii and M. Ohtani, Phys. Rev. D 70, 014016 (2004).
[15] D. T. Son and M. A. Stephanov, Phys. Rev. D 70, 056001 (2004).

[16] Y. Minami, Phys. Rev. D 83, 094019 (2011).

[17] P. C. Hohenberg and B. I. Halperin, Rev. Mod. Phys. 49, 435 (1977).

[18] M. G. Alford, A. Schmitt, K. Rajagopal, and T. Schäfer, Rev. Mod. Phys. 80, 1455 (2008).

[19] P. M. Chaikin and T. C. Lubensky, Principles of Condensed Matter Physics (Cambridge University Press, Cambridge, England, 1995).

[20] S. Weinberg, The Quantum Theory of Fields, Modern applications Vol. 2 (Cambridge University Press, Cambridge, England, 1996).

[21] D. T. Son, hep-ph/0204199.

[22] I. E. Dzyaloshinskii and G. E. Volovick, Ann. Phys. (N.Y.) 125, 67 (1980).

[23] M. A. Stephanov, K. Rajagopal, and E. V. Shuryak, Phys. Rev. Lett. 81, 4816 (1998); Phys. Rev. D 60, 114028 (1999). 